\title{
Erratum to: Identification and characterization of $E Y K 1$, a key gene for erythritol catabolism in Yarrowia lipolytica
}

\author{
F. Carly ${ }^{1,2}$ • H. Gamboa-Melendez ${ }^{2}$ - M. Vandermies ${ }^{3}$ - C. Damblon ${ }^{4}$ - J. M. Nicaud ${ }^{2}$. \\ P. Fickers ${ }^{3}$
}

Published online: 12 July 2017

(C) Springer-Verlag GmbH Germany 2017

\section{Erratum to: Appl Microbiol Biotechnol DOI 10.1007/s00253-017-8361-y}

The original article was updated because several pieces of information were omitted. The following paragraph has been added in section Material and methods:

\section{Sequences analysis}

Genome sequences of Yarrowia species were assembled and annotated by Cécile Neuvéglise, Hugo Devillers and coworkers (to be published). Homologs of EYK1 in Yarrowia species were identified by Blast on the private site of GRYC (Genome Resources for Yeast Chromosomes;

The online version of the original article can be found at http://dx.doi.org/ 10.1007/s00253-017-8361-y

P. Fickers

pfickers@ulg.ac.be

1 Unité de Biotechnologies et Bioprocédés, Université Libre de Bruxelles, Brussels, Belgium

2 Micalis Institute, INRA, AgroParisTech, Université Paris-Saclay, 78350 Jouy-en-Josas, France

3 Microbial Processes and Interactions, TERRATeaching and Research Centre, University of Liège - Gembloux Agro-Bio Tech, Gembloux, Belgium

4 Laboratoire de Chimie Biologique Structurale, Département de Chimie, Université de Liège, Liège, Belgium http://gryc.inra.fr) using EYK1 gene as template and retrieved using the download functionality developed by H. Devillers.

The legend of Fig. S1 should be read as follows:

Multiple alignments of YALYOF01606g genes with the $D A K 1, D A K 2$ and DAK3 in the lipolytica clade. Homologs of EYK1 in Yarrowia species were identified by Blast on the private site of GRYC using EYK1 gene as template and retrieved using the download functionality. Color code: red, similar in all gene; green, similar in two of the three genes; yellow highlighted, different in DAK3 group compared to DAK1 and DAK2 gene. Sequences are from strains YALI: Yarrowia lipolytica CLIB122; YAGA: Yarrowia galli CBS 9722 (96.77\%); YAYA: Yarrowia yakushimensis CBS 10253 (91.62\%); YAAL: Yarrowia alimentaria CBS 10151 (87.22\%) and YAPH: Yarrowia phangngensis CBS 10407 (85.01\%). Maximal identities with Yarrowia lipolytica EYK1 (YALI0F01606g) are indicated in bracket.

The following information was missing in the Acknowledgements section:

The genome sequence of $Y$. galli was funded by the project CALIN (Carburants Alternatifs et Systèmes d'Injection, grant $\mathrm{N}^{\circ}$ 25331). The genomes of Y. yakushimensis, Y. alimentaria, and $Y$. phangngensis were funded by INRA in the frame of the AIP-Bioressources 2011 program (YALIP project granted to C. Neuvéglise). 